\title{
Camber effects in the dynamic aeroelasticity of compliant airfoils
}

\author{
J. Murua, R. Palacios*, J. Peiró \\ Department of Aeronautics, Imperial College, London SW7 2AZ, United Kingdom
}

\begin{abstract}
This paper numerically investigates the effect of chordwise flexibility on the dynamic stability of compliant airfoils. A classical two-dimensional aeroelastic model is expanded with an additional degree of freedom to capture time-varying camber deformations, defined by a parabolic bending profile of the mean aerodynamic chord. Aerodynamic forces are obtained from unsteady thin airfoil theory and the corresponding compliant-airfoil inertia and stiffness from finite-element analysis. V-g and state-space stability methods have been implemented in order to compute flutter speeds. The study looks at physical realizations with an increasing number of degrees of freedom, starting with a camber-alone system. It is shown that single camber leads to flutter, which occurs at a constant reduced frequency and is due to the lock in between the shed wake and the camber motion. The different combinations of camber deformations with pitch and plunge motions are also studied, including parametric analyses of their aeroelastic stability characteristics. A number of situations are identified in which the flutter boundary
\end{abstract}

\footnotetext{
*Corresponding author. Tel.: +44 20759 45075; fax: +44 2075848120.

Email address: rpalacio@imperial.ac.uk (R. Palacios)
} 
of the compliant airfoil exhibits a significant dip with respect to the rigid airfoil models. These results can be used as a first estimation of the aeroelastic stability boundaries of membrane-wing micro air vehicles.

Key words: Conformable Airfoils, Camber Deformations, Flutter, Aeroelastic Stability

\section{Nomenclature}

$\begin{array}{ll}a & \text { coordinate of elastic axis in semi-chords } \\ b & \text { semi-chord } \\ c_{L} & \text { lift coefficient, per unit span } \\ c_{M_{a b}} & \text { moment coefficient about the elastic axis, per unit span } \\ c_{\Lambda} & \text { bimoment coefficient, per unit span } \\ C(k)=F(k)+i G(k) & \text { Theodorsen's wake function } \\ E & \text { elastic modulus of airfoil's material } \\ g_{i} & \text { (fictitious) modal structural damping } \\ h(x, t) & \text { airfoil deformation } \\ I_{\alpha}=\int[(x-a b) \alpha]^{2} d A & \text { airfoil moment of inertia around elastic axis } \\ I_{\delta}=\int[\Psi(x)]^{2} d A & \text { airfoil camber moment of inertia } \\ k=\frac{\omega b}{V} & \text { reduced frequency } \\ K_{\alpha} & \text { torsional stiffness of airfoil around elastic axis, per unit span } \\ K_{\delta} & \text { camber stiffness, per unit span } \\ K_{\zeta} & \text { plunge stiffness, per unit span } \\ L & \text { lift, per unit span } \\ L_{n} & \text { generalized forces }\end{array}$




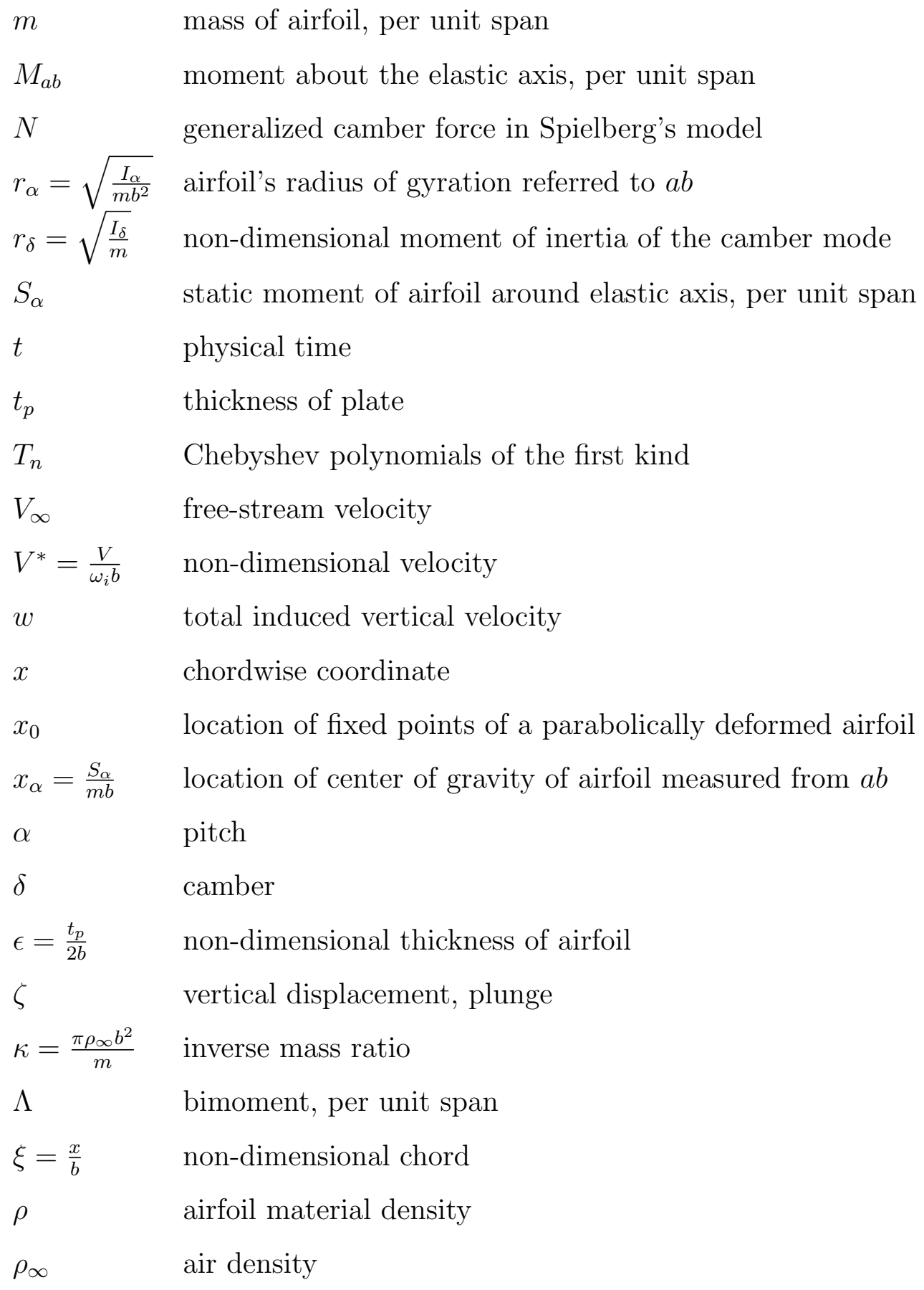




$$
\begin{array}{ll}
\Psi(x) & \text { camber shape } \\
\omega & \text { oscillation frequency } \\
\omega_{\alpha}=\sqrt{\frac{K_{\alpha}}{I_{\alpha}}} & \text { characteristic frequency of pitch mode } \\
\omega_{\delta}=\sqrt{\frac{K_{\delta}}{I_{\delta}}} & \text { characteristic frequency of camber mode } \\
\omega_{\zeta}=\sqrt{\frac{K_{\zeta}}{m}} & \text { characteristic frequency of plunge mode }
\end{array}
$$

\section{Subscripts}

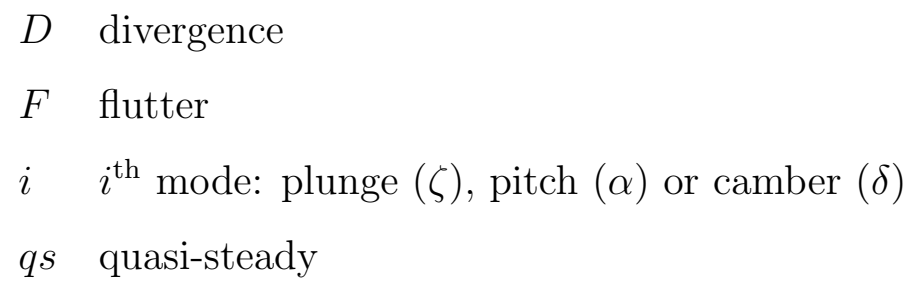

\section{Introduction}

Advances in the technology of smart actuators and compliant structures have finally made conformable wings a realizable goal. As aerodynamic contours can be continuously optimized, wings with actively-controlled compliant airfoils can be designed to be more efficient than those with discrete flaps. The idea however is certainly not new, and the first reported mechanism to change airfoil geometry can be traced back to the work of Parker (1920), who proposed a variable-camber rib as a high-lift device for take-off and landing. Examples of current applications under study include generic conformable leading edge (Santer and Pellegrino, 2007) and trailing edge (Bak et al., 2007) concepts, piezoelectric-actuated active twist rotors for noise and vibration reduction in rotary wings (Cesnik et al., 2001; Bernhard and Chopra, 2001), deformable airfoils for load alleviation in wind turbines (Barlas and van Kuik, 2007) and aeroelastic amplification of high-lift devices in fixed wings (Cam- 
panile and Anders, 2005). Moreover, actuation through variable camber is expected to require less power than conventional flaps for a given control authority (Johnston et al., 2007). Adaptive wing technologies are also expected to play a key role in the successful development of flapping-wing micro air vehicles, in such critical aspects as maneuverability (Stanford et al., 2007) or control of flow separation (Katam et al., 2005). Concepts for dynamic stall control in rotary wings using compliant airfoil concepts have also been proposed (Kerho, 2007). Nature is the source of inspiration in many of these applications, as the extremely high energy efficiency of natural fliers and swimmers is achieved with highly deformable surfaces (Lentink et al., 2007). In fact, the classical models used to describe the biomechanics of fish propulsion, developed by Lighthill (1960) and Wu (1971), present many similarities to those used to estimate the aeroelastic response of compliant wings.

Wing adaptation is obtained in part by a reduction of stiffness in the wing primary structures, and this may also have an undesirable impact in the aeroelastic stability characteristics. In this context, this paper presents a basic procedure to introduce the effect of airfoil flexibility in first estimations of the flutter velocity. The starting point is the 2-D aeroelastic model first proposed by Theodorsen (1935) for the estimation of flutter speeds in slender wings. It assumes, as it is also done in many related formulations that have been proposed thereafter, that the structural characteristics of the wing can be approximately represented by a beam with rigid cross sections. Theodorsen's solution relies on a closed-form analytical solution of the thinairfoil unsteady aerodynamics problem in the frequency domain. Based on the indicial response method, Wagner (1925) had proposed the equivalent 
time-domain solution, which sets the basis for state-space formulations of the problem, such as those of Leishman and Nguyen (1989) and Peters et al. (1995). An extension to Theodorsen's solution for arbitrary boundary conditions was first developed by Sears (1941) to study continuous gust response. The unsteady lift corresponding to parabolic bending deformations was originally proposed by Spielberg (1953) and general deformations of the airfoil were later introduced by $\mathrm{Wu}$ (1961) in the analysis of fish swimming propulsion. Based on Spielberg's approach, Rodden and Stahl (1969) formulated the flutter problem for small-aspect-ratio wings.

Despite the current widespread use of panel methods in unsteady aerodynamics, 2-D aerodynamic models are still used via strip theory to obtain a first estimate of the flutter stability boundary on slender and rotary wing problems. In the context of rotary-wing applications, the transient 2-D aerodynamic loads due to time-dependent airfoil deformations have been recently studied in state-space form by Gaunaa (2006) and Peters (2008), including the evaluation of the bimoments associated to the airfoil chordwise bending deformations. Palacios and Cesnik (2008a) coupled those aerodynamic models with an extended beam analysis to provide a low-order description of the dynamic of large-aspect-ratio wings with compliant airfoils. Of course, it is always possible to solve the 2-D potential-flow equations with arbitrary boundary conditions using numerical discretization. This has been the approach in numerous studies on flutter of cantilever plates and membranes for applications in problems ranging from human snoring (Huang, 1995) to flag dynamics (Argentina and Mahadevan, 2005). Tang et al. (2009) have recently presented an exhaustive numerical investigation of the dynamics of 
this system, including the nonlinear behavior at postcritical velocities. The aim of this work is however to obtain analytical expressions that describe the main physical phenomena in the dynamic response of wings with deformable airfoils. Chordwise flexibility is allowed, but limited to small changes of camber. This will allow a quick estimation of the stability boundaries and the identification of the main interactions in the flutter mechanism.

To that goal, this paper follows a methodology similar to that presented in (Palacios and Cesnik, 2008a) for static aeroelastic equilibrium. Chordwise flexibility is defined by a prescribed parabolic shape function, and the corresponding stiffness is obtained via conventional finite-element analysis for the actual wing geometry. Palacios and Cesnik (2008b) presented a numerical procedure for the estimation of the camber stiffness using asymptotic approximations, but here a standard finite-element package is chosen to make the methodology easily accessible. The unsteady aerodynamic model is based on the solution process introduced by Peters (2008) for arbitrary airfoil deformations, with aerodynamic lags obtained as in (Leishman and Nguyen, 1989). This procedure defines a simple state-space model for the airfoil dynamics, for which linear stability studies can be easily carried out thus giving the main parameter dependencies of the flutter boundary. A system with three physical degrees of freedom (DOF) is considered, corresponding to two rigid-body (plunge and pitch motions) and one elastic (camber deformation) DOF. The resulting description is somehow similar to that introduced by Theodorsen and Garrick (1941) to explain aileron flutter, but with a different physical meaning of the additional degree of freedom in the problem. The chordwisebending degree of freedom introduced here will be associated to a instability 
mechanism that will be referred to as "camber flutter".

\section{Theoretical formulation}

The system subject to study is shown in Figure 1. A thin airfoil, initially located in $[-b, b]$ along the $x$ axis and under a uniform $V_{\infty}$ free-stream is assumed. Three DOF are considered: plunge, $\zeta$, pitch, $\alpha$, and camber, $\delta$. The physical realization of this system is also represented in Figure 1. The airfoil is attached to two linear springs, with stiffness constants $K_{1}$ and $K_{2}$, at two points located at a distance $d / 2$ from the mid-chord point. Both springs are equivalent to a translational and a torsional spring, of stiffness $K_{h}=K_{1}+K_{2}$ and $K_{\alpha}=\frac{K_{1} K_{2}}{K_{1}+K_{2}} d^{2}$, respectively, co-located at a distance $a b=\frac{K_{2}-K_{1}}{K_{1}+K_{2}} \frac{d}{2}$ aft the airfoil mid-chord. This point defines the elastic axis. The deflection of an airfoil with general chordwise deformation can then be written as

$$
h(x, t)=\zeta(t)+(x-a b) \alpha(t)+\Psi(x) \delta(t),
$$

where $\zeta$ is the vertical displacement (positive down) of the elastic axis and $\Psi(x)$ represents the assumed camber shape. So as to account for the firstorder elastic effects, it will be assumed that

$$
\Psi(x)=\left(\frac{x}{b}\right)^{2}-\frac{1}{3} .
$$

This camber deformation accounts for the next term ( $2^{\text {nd }}$ order) in a

Taylor series expansion of airfoil deformations. The $\frac{1}{3}$ comes from imposing orthogonality of the different DOF with respect to the area integral (Palacios 
and Cesnik, 2008b). The equations of motion of the system shown in Figure 1 can be expressed, using non-dimensional coefficients, as

$$
\begin{aligned}
\frac{\ddot{\zeta}}{b}+x_{\alpha} \ddot{\alpha}+\omega_{\zeta}^{2} \frac{\zeta}{b}+\Psi(a b) \omega_{\delta}^{2} \frac{\delta}{b} & =\frac{\kappa}{\pi}\left(\frac{V_{\infty}}{b}\right)^{2} c_{L}, \\
x_{\alpha} \frac{\ddot{\zeta}}{b}+r_{\alpha}^{2} \ddot{\alpha}+\left(r_{\alpha} \omega_{\alpha}\right)^{2} \alpha & =2 \frac{\kappa}{\pi}\left(\frac{V_{\infty}}{b}\right)^{2} c_{M_{a b}}, \\
r_{\delta}^{2} \frac{\ddot{\delta}}{b}+\Psi(a b) \omega_{\zeta}^{2} \frac{\zeta}{b}+\left\{\left(r_{\delta} \omega_{\delta}\right)^{2}+\left[\Psi(a b) \omega_{\zeta}\right]^{2}\right\} \frac{\delta}{b} & =\frac{\kappa}{\pi}\left(\frac{V_{\infty}}{b}\right)^{2} c_{\Lambda} .
\end{aligned}
$$

where the classical aeroelastic nomenclature has been followed. For the par-

ticular case of a thin homogeneous plate, it is $r_{\delta}^{2}=\frac{4}{45}, r_{\alpha}^{2}=\frac{1}{3}+a^{2}$ and $x_{\alpha}=-a$.

\subsection{Structural model}

In order to estimate the camber deflection stiffness of the airfoil, a finiteelement model of an isotropic cantilever thin plate has been built in ABAQUS (1998). As the interest focuses in the internal solution of the problem (in a Saint-Venant sense), models with increasing aspect ratios were tested until a converged value has been obtained. Planar (S4R) shell elements have been used, obtaining the response to distributed bimoments. In particular, the cross-sectional loads of Figure 2 were investigated, as in (Palacios and Cesnik, 2008b). If $p(x)$ are pressure loads applied along the airfoil camberline, the corresponding generalized camber force per unit span length is defined as

$$
\Lambda=\int_{-b}^{b} p(x) \Psi(x) d x .
$$

In particular, for the applied bimoments defined in Figure 2, the generalized camber force is given by $\Lambda=2 F$ and the chordwise bending stiffness 
is determined from the ratio between the generalized camber force, $\Lambda$, and deformation, $\delta$, as

$$
K_{\delta}=\frac{\Lambda}{\delta}=\frac{2 F}{\delta}
$$

where $\delta$ is obtained by curve-fitting the parabolic shape of the deformed plate. Computed results for a linear elastic material with a Poisson's ratio of $\nu=0.3$ are shown in Figure 3. The camber stiffness is normalized as $\frac{K_{\delta}}{E \epsilon^{3}}$, where $E$ is the material's elastic modulus and $\epsilon=\frac{t_{p}}{2 b}$ is the non-dimensional thickness, $t_{p}$ representing the thickness of the plate. The values of Figure 3 will be used in what follows, but this procedure is equally applicable to different airfoil geometries and properties.

\subsection{Aerodynamic model}

The starting point to compute the aerodynamic loads in the system of Figure 1 is the 2-D finite-state formulation for flexible airfoils by Peters (2008). Although the frame of reference can have arbitrarily large motions, the de-

formations of the airfoil are assumed to be small, so that $h \ll b, \frac{\partial h}{\partial x} \ll 1$, and $\frac{\partial h}{\partial t} \ll V_{\infty}$. For the same reason, the trailing edge vorticity is assumed to be shed along the $x$-axis.

The flow-tangency boundary condition is expressed as

$$
w=V_{\infty} \frac{\partial h}{\partial x}+\frac{\partial h}{\partial t},
$$

where $w$ is the total induced vertical velocity, comprising contributions from wake vorticity and bound circulation. Aerodynamic loads can be obtained 
by Glauert's method of expanding all variables in terms of Chebyshev polynomials (Peters et al., 1995). The generalized forces are defined as

$$
L_{n}=-b \int_{-1}^{1} T_{n}(\xi) \Delta P d \xi,
$$

where $T_{n}(\xi)$ are the Chebyshev polynomials of the first kind (Abramowitz and Stegun, 1972) along the non-dimensional direction, $\xi=\frac{x}{b} \in[-1,1]$. Assuming harmonic oscillations, Theodorsen's formulation is recovered and the generalized forces are given by Palacios and Cesnik (2008a), as

$$
\begin{aligned}
\frac{L_{0}}{\pi \rho b} & =-b\left(\ddot{\zeta}-a b \ddot{\alpha}-\frac{1}{12} \ddot{\delta}+V_{\infty} \dot{\alpha}\right)-2 V_{\infty} C(k)\left(\alpha_{q s}+\delta_{q s}\right), \\
\frac{L_{1}}{\pi \rho b} & =-\frac{b^{2}}{8} \ddot{\alpha}-\frac{V_{\infty} b}{2} \dot{\alpha}-\frac{V_{\infty}}{2} \dot{\delta}-\frac{V_{\infty}^{2}}{2} \delta+V_{\infty} C(k)\left(\alpha_{q s}+\delta_{q s}\right), \\
\frac{L_{2}}{\pi \rho b} & =\frac{b}{2} \ddot{\zeta}-\frac{a b^{2}}{2} \ddot{\alpha}-\frac{b}{12} \ddot{\delta}+V_{\infty} b \dot{\alpha}+\frac{V_{\infty}^{2}}{b} \delta,
\end{aligned}
$$

where $C(k)$ represents Theodorsen's complex wake function and $k=\frac{\omega b}{V_{\infty}}$ corresponds to the reduced frequency. The symbols $\alpha_{q s}$ and $\delta_{q s}$ stand for the quasi-steady angle of attack and camber deformation, respectively, and are given by

$$
\begin{aligned}
\alpha_{q s} & =\dot{\zeta}+\left(\frac{1}{2}-a\right) b \dot{\alpha}+V_{\infty} \alpha, \\
\delta_{q s} & =\frac{1}{6} \dot{\delta}+\frac{V_{\infty}}{b} \delta .
\end{aligned}
$$

Finally, the generalized aerodynamic loads can be written as

$$
L=\int_{-b}^{b} \Delta P d x=-L_{0}
$$




$$
\begin{aligned}
M_{a b} & =-\int_{-b}^{b} \Delta P(x-a b) d x=b L_{1}-a b L_{0}, \\
\Lambda & =-\int_{-b}^{b} \Delta P\left[\left(\frac{x}{b}\right)^{2}-\frac{1}{3}\right] d x=\frac{1}{2} L_{2}+\frac{1}{6} L_{0} .
\end{aligned}
$$

\subsection{Methods for stability analysis}

The dynamic stability of the system defined in Eq. (3) will be studied both in frequency (V-g method) and time domain (using indicial response method to obtain a state-space approximation of the unsteady aerodynamic forces). This work follows the methodology proposed by Leishman and Nguyen (1989), among others. In this case, Wagner's function, i.e., the Fourier transform of Theodorsen's function, $C(k)$, is approximated by an exponential function and the state-space equations are obtained by direct application of Laplace transforms to the indicial response. In this process two new aerodynamic states arise for each quasi-steady DOF, given by Eqs. (9) and (10), which are contained in the state-vector. The resulting firstorder ordinary differential equations describing the unsteady aerodynamics can be appended to the structural dynamics equations governing the airfoil motion, Eq. (3). The eigenvalues of the resulting dynamics matrix determine directly the stability of the system.

In this work, the state-space approach has been found to be computationally faster, but the V-g and V- $\omega$ plots of the V-g method provide extra information to interpret the results.

\section{Comparison with other studies}

As a first step, in order to validate the methods for calculating the aerodynamic loads, the values obtained using Eqs. (8-11) are compared to those 
published by Spielberg (1953), who studied the parabolic bending profile of the mean chord line and examined it against a $50 \%$ control surface. The definition of the camber DOF differs from the one used here, but, as it will be seen, both theories are equivalent.

Figure 4 shows Spielberg's model, where the subscript $S$ is used to distinguish the DOF from those of Figure 1. In this case, plunge is measured at the mid-chord, i.e., $a=0$. The airfoil's deflection is given by

$$
h(x, t)=\zeta_{S}(t)+x \alpha_{S}(t)+\left[1-\left(\frac{x}{b}\right)^{2}\right] \delta_{S}(t) .
$$

Comparison of Eqs. (1) and (12) for $a=0$ provides the relationship between the DOF in each model as

$$
\zeta_{S}=\zeta+\frac{2}{3} \delta, \quad \alpha_{S}=\alpha, \quad \delta_{S}=-\delta .
$$

On the other hand, the generalized camber force is given by

$$
N=-\int_{-b}^{b} \Delta P\left[1-\left(\frac{x}{b}\right)^{2}\right] d x=\frac{L_{2}-L_{0}}{2} .
$$

Substituting Eq. (13) into Eqs. (8-11) with $a=0$, Spielberg's model is recovered. Furthermore, if the generalized camber force given by Eq. (14) is considered instead of the bimoment $\Lambda$ defined in Eq. (11), the expressions for the aerodynamic loads are identical to those published by Spielberg (1953).

The aerodynamic loads given by Eq. (11) have been been also compared to results obtained using an in-house implementation of the unsteady vortexlattice method (UVLM) (Katz and Plotkin, 2001), also based on potential flow theory and thin-airfoil assumptions. Using a panel-like discretization, elementary solutions are distributed over a surface and the non-penetration 
boundary condition is enforced at a number of control points. From the pressure distribution obtained for prescribed kinematics, the camber bimoment can then be calculated using Eq. (4). Here, chordwise harmonic deflections have been prescribed, with the assumed camber mode of Eq. (2) and an amplitude of $1 \%$ of the chord. A very high-aspect-ratio wing has been assumed in the UVLM, $R=100$, with 10 panels along the spanwise direction. The number of chordwise panels is inversely proportional to the time-step, so to capture the unsteadiness of the flow and to assure a good representation of the camber shape, the most restrictive condition has been enforced, requiring a minimum of 210 sampling points per cycle and 20 chordwise panels. The rollup of the wake has been disabled and the amplitude of the deformations is small enough to guarantee an near-flat wake, comparable to the analytical model based on Theodorsen's flat wake assumption.

The aerodynamic transfer function has been considered for verification purposes, defined as the quotient between the Fourier transform of the timedomain aerodynamic quantity of interest and the camber motion. Figures 5 and 6 depict, respectively, the values of the modulus and argument of the transfer functions for lift and bimoment coefficients per unit span. The agreement is remarkable and has served to verify the implementation of the aerodynamic model used in this work.

Next, the numerical methods to compute the instability onset have been validated. Although there is not available data to compare flutter speeds for a cambered airfoil, the algorithms can be validated for the classical plungeand-pitch airfoil, since the addition of new DOF to the system requires just a natural extension of the algorithm. Therefore, results have been compared to 
the study published by Zeiler (2000), where he found that a number of plots published by Theodorsen and Garrick (1941) were in error - this discrepancy has also been recently reported (Bergami and Gaunaa, 2009). As shown in Figure 7, the agreement with Zeiler is excellent.

\section{Numerical results}

In order to determine the stability boundaries of an airfoil with chordwise deformations and to elucidate the mechanisms that lead to flutter under different conditions, the results obtained by the V-g method have been complemented with tools such as $\mathrm{V}-\mathrm{g} / \mathrm{V}-\omega$ graphs and eigenvector analysis.

For the system shown in Figure 1, the parameters that univocally determine the flutter problem are: the location of the elastic axis, $a$, the inverse mass ratio, $\kappa$, and the frequency ratios between DOF, i.e., $\frac{\omega_{\zeta}}{\omega_{\alpha}}$, and $\frac{\omega_{\delta}}{\omega_{\alpha}}$.

The analysis follows an increasing complexity approach, starting with a single-DOF system (camber). Then, non-classical combinations of two DOF are studied, namely, plunge-camber and pitch-camber. Finally, the system comprising the three modes is considered.

\subsection{One degree of freedom: camber deformations}

Consider first a single camber degree of freedom on a thin plate. Figure 8 illustrates the physical system, where the plate is fixed at two symmetrically located points, at distances $\pm x_{0}$ from the mid-chord, with $x_{0}=\frac{b \sqrt{3}}{3}$. This corresponds to the position of the linear springs in the physical realization of the system shown in Figure 1. This value is due to the assumed parabolic bending mode, Eq. (2). It is further assumed that the elastic axis of the homogeneous flat plate coincides with the mid-chord, i.e., $a=0$. 
Introducing the unsteady aerodynamic camber bimoment, $\Lambda$, given by Eq. (11), and removing all dependencies with respect to other DOF in Eq. (3), the stability equation in the camber DOF only is

$$
-\omega^{2}\left(1+\frac{\kappa}{36 r_{\delta}^{2}}\right)+i \omega \frac{\kappa}{18 r_{\delta}^{2}} \frac{V_{\infty}}{b} C(k)+\left\{\omega_{\delta}^{2}-\frac{\kappa}{r_{\delta}^{2}}\left[\frac{1}{2}-\frac{1}{3} C(k)\right]\left(\frac{V_{\infty}}{b}\right)^{2}\right\}=0 .
$$

Recall that $r_{\delta}^{2}=\frac{4}{45}$ for the homogeneous thin plate subject to study in this case. The flutter speed can be analytically obtained by zeroing the real and imaginary parts of this equation. It occurs at a constant reduced frequency, irrespective of the rest of the parameters of the problem, given by

$$
k_{F}=-\left[\frac{1}{2}-\left(\frac{x_{0}}{b}\right)^{2}\right]^{-1} \frac{G\left(k_{F}\right)}{F\left(k_{F}\right)}=-6 \frac{G\left(k_{F}\right)}{F\left(k_{F}\right)} \approx 1.07,
$$

where $F(k)$ and $G(k)$ stand, respectively, for the real and imaginary parts of Theodorsen's function, i.e., $C(k)=F(k)+i G(k)$. The ratio $\frac{G(k)}{F(k)}$ is related to the phase shift between the quasi-steady and the circulatory aerodynamic loads (Bisplinghoff et al., 1955). This implies that dynamic instability occurs as the shed wake locks in with the camber motion.

Classically, flutter has been analyzed in systems with at least two DOF, where the instability is due to the interchange of energy between plunge and pitch oscillation modes (Theodorsen, 1935). However, even though singleplunge is an inherently stable mode, an airfoil with single pitch oscillations can become unstable if the elastic axis is close enough to the leading edge (for values of $a \lesssim-0.68$ ). Likewise, the camber degree of freedom does not require any other mode for flutter to occur; it is the interaction between the wake and the airfoil what causes instability. This is further corroborated by 
a quasi-steady analysis (ignoring wake dynamics), which yields no flutter at all.

Similar conclusions were obtained by Huang (1995), who studied the flutter of finite flexible cantilevered plates in axial flow on the context of human snoring. Following energetic considerations, he showed that the noncirculatory part of the aerodynamic loads does not contribute to flutter, since the work done over a cycle is negligible. Conversely, the circulatory part does positive work and it is key to flutter. In fact, for a flexible plate in a free stream, there will be a flow velocity that will excite a resonant camber bending mode (Argentina and Mahadevan, 2005).

The constant value of reduced frequency at which instability occurs leads to a non-dimensional flutter velocity that only depends on the inverse mass ratio, $\kappa$,

$$
V_{F}^{*}=\frac{V_{F}}{\omega_{\delta} b}=\frac{1}{\sqrt{k_{F}^{2}+c\left(k_{F}\right) \kappa}},
$$

where $c\left(k_{F}\right)$ only depends on the (constant) reduced frequency obtained from Eq. (16), and is given by

$$
c\left(k_{F}\right)=\frac{1}{r_{\delta}^{2}}\left\{\left[\frac{G\left(k_{F}\right)}{F\left(k_{F}\right)}\right]^{2}-\frac{1}{3} \frac{\left\|C\left(k_{F}\right)\right\|^{2}}{F\left(k_{F}\right)}+\frac{1}{2}\right\} .
$$

On the other hand, for this 1-DOF system, the divergence speed is given by

$$
V_{D}^{*}=\frac{V_{D}}{\omega_{\delta} b}=\sqrt{\left[\frac{1}{2}-\left(\frac{x_{0}}{b}\right)^{2}\right]^{-1} \frac{r_{\delta}^{2}}{\kappa}} .
$$

Substituting numerical values in Eqs. (17) and (19), it is found that the 
flutter speed $V_{F}^{*} \approx \sqrt{\frac{1}{1.14+3.91 \kappa}}$ is smaller than the divergence speed $V_{D}^{*}=$ $\sqrt{6 \frac{r_{\delta}^{2}}{\kappa}}$, for any value of $\kappa$. This is also consistent with Huang (1995), who experimentally verified the absence of static divergence for a finite flexible plate.

\subsection{Two degrees of freedom: plunge-camber and pitch-camber}

Combinations of two DOF are studied next with the elastic axis located at the airfoil mid-chord point $(a=0)$. First, a system defined by plunge and camber is considered, as depicted in Figure 9. A second system with two DOF is defined by pitch and camber motions, as illustrated in Figure 10.

Figures 11 and 12 show the stability boundaries for these 2-DOF systems, as a function of the frequency ratio and for different values of the inverse mass ratio, $\kappa$. In this case, a $0.5 \%$ numerical damping has been included in order to eliminate mild flutter or neutral stability points. Flutter speed for the camber-only system, Eq. (17), is also presented in both cases to help interpret results.

Figure 11 presents the results obtained for the stability boundary of the plunge-camber system of Figure 9, compared to the single-camber system of Figure 8. It can be inferred that flutter, in this system, is a camber-driven phenomena (camber-wake interaction), and the inclusion of the plunge DOF is stabilizing - recall that, as mentioned above, plunge alone is a stable mode.

Note that the plunge-camber system illustrated in Figure 9 exhibits coupling, both structural and aerodynamic. Hence, the natural frequencies of the system are not the same as the characteristic frequencies defined for each single mode. 
The pitch-camber airfoil shown in Figure 10 exhibits a behavior that can be divided into three main zones (Figure 12):

(i) In the first one, for approximately $\frac{\omega_{\delta}}{\omega_{\alpha}} \lesssim 0.4$, flutter is governed by camber. The flutter curve of pitch and camber follows exactly the curve obtained for the single-DOF system in camber deformations. Hence, no coupling between modes occurs. Physically, this region corresponds to a relatively high stiffness in twist.

(ii) In the second region, $0.4 \lesssim \frac{\omega_{\delta}}{\omega_{\alpha}} \lesssim 1.4$, the curve of two DOF departs from the camber-alone counterpart, due to the interaction of pitch and camber modes. This coupling plays a stabilizing role compared to camber induced flutter, clearly steeping the curve. This stabilizing effect, in turn, diminishes as the inverse of the mass parameter, $\kappa$, increases. This 2-DOF flutter comprises the region in which the characteristic frequencies of both modes are close.

(iii) At higher frequency ratios, $\frac{\omega_{\delta}}{\omega_{\alpha}} \gtrsim 1.4$, static divergence occurs first. Response is then dominated by the pitch DOF, since beyond this point, the twist stiffness is low compared to the chordwise stiffness.

\subsection{Three degrees of freedom}

For the 3-DOF problem, Figure 13 shows the flutter speed surfaces as a function of the frequency ratios $\frac{\omega_{\delta}}{\omega_{\alpha}}$ and $\frac{\omega_{\zeta}}{\omega_{\alpha}}$, for $a=0$ and $\kappa=0.2$.

The stability boundaries shown in Figure 13 were checked to be representative for values of $a$ and $\kappa$ satisfying $a \geq 0$ and $\kappa \in[0.05,0.5]$. In order to make it easier to visualize, Figure 14 shows a relevant 2-D curve of the 3-DOF system compared to simpler systems with two DOF (plunge-camber 
and pitch-camber). This distinctive 2-D curve corresponds to the frequency ratio $\frac{\omega_{\zeta}}{\omega_{\alpha}}=1$ (dashed line in Figure 13). Note that for the set of parameters considered here, the 2-DOF plunge-pitch classical system does not exhibit flutter.

Four main regions have been identified, denoted by A, B, C and D. Comparing the flutter curves for these three different systems and aided by V-g and V- $\omega$ plots, the flutter mechanisms in these regions can be inferred as follows:

(i) Region A. At low enough $\frac{\omega_{\delta}}{\omega_{\alpha}}$ (i.e., very large torsional stiffness), flutter is dominated by the camber mode. Due to the camber-plunge coupling, it is a 2-DOF phenomena - similar to the previously studied case (Figure 11). As shown in the V-g plot (Figure 15), the pitch mode is always stable and does not contribute to flutter. Recall that in the absence of camber (classical plunge-pitch 2-DOF system), there is no flutter under this parameter combination. The V-g plot also shows that the inclusion of camber significantly alters the damping of the plunge mode.

(ii) Regions $B$ and $C$. There is a point at around $\frac{\omega_{\delta}}{\omega_{\alpha}} \approx 0.1$, where plungecamber and plunge-pitch-camber curves split and a new mechanism drives flutter, where the three modes interact together. Figures 17 and 18 show, respectively, typical V-g and V-w plots of region C, which is analogous to region B. The main difference between V-g plots of Figures 15 and 17 is that, at flutter onset, the damping curve of pitch is still above the plunge curve in the latter, so this mode cannot be ignored in this case. Furthermore, it can be seen that there is plunge and pitch vibration-frequency coalescence near the flutter speed, but it 
is the camber DOF which eventually becomes unstable. Hence, there is a complex interaction among the three modes. Note also that, again, the inclusion of camber makes the 3-DOF system unstable, in contrast to the classical plunge-pitch 2-DOF system (stable under this set of parameters). In regions $\mathrm{B}$ and $\mathrm{C}$, the characteristic frequencies of all three modes are of the same order of magnitude, so this behavior can be expected.

(iii) Region $D$. In this case, pitch dominated static divergence occurs before dynamic instability, since it corresponds to a very low stiffness of this mode.

The eigenvectors of each mode at the flutter point have been also computed for the different regions and are shown in Table 1. It can be observed that the amplitude of the pitch mode is negligible compared to the other two modes in region A. Hence, it does not affect flutter, which as aforementioned, is a 2-DOF phenomenon here. In regions $\mathrm{B}$ and $\mathrm{C}$, the vibration modes lag each other by, approximately, $90^{\circ}$ and they differ in amplitude, at most, by one order of magnitude. This proves the triple interaction mechanism that drives flutter.

Finally, the effect of the position of the elastic axis on the dynamic stability of the 3-DOF aeroelastic system (Figure 1) has been analyzed. Figure 19 shows a comparison of positive and negative values of $a$, representative of the whole range.

It can be inferred from Figure 19 that a positive value of $a$ (elastic axis aft) simply scales and displaces the flutter curve. Conversely, for $a<0$ (elastic axis fore) the consequences are significant, since the characteristic trough is 
completely removed. Hence, small values of flutter speed are avoided and in fact, the closer the elastic axis is to the leading edge, the larger the stability envelope - in contrast to a single-pitch system.

\section{Concluding remarks}

It has been shown that a first estimate of the aeroelastic stability characteristics of a flexible airfoil can be analytically determined if the conventional pitch-and-plunge aeroelastic model is expanded with a new DOF that measures the instantaneous averaged camber deformation. This paper has proposed a description of this problem and a solution methodology. In the proposed approach, the camber stiffness is obtained by a finite-element analysis and the unsteady aerodynamic loads from a finite-state model of a thin airfoil.

Expressions for the critical speed and frequency have been obtained, and results have been interpreted in order to elucidate the parameters that affect the onset of flutter. The stability analysis of the resulting 2-D aeroelastic model provides the simplest possible way to predict "camber flutter" and has helped identifying its dominant physical mechanisms. This expands the work of Palacios and Cesnik (2008a), that from similar models studied the static aeroelastic equilibrium conditions for compliant wings. Moreover, this work has focused on the 2-D airfoil problem and has removed the need for the extended beam model introduced in Palacios and Cesnik (2008b). Instead, a simpler numerical procedure based on conventional finite-element analysis has been introduced to obtain the stiffness and inertia characteristics of deformable airfoils. 
It has been found that camber mode alone can lead to linear flutter. The instability always occurs at the same reduced frequency, independently of the rest of parameters in the problem. The flutter mechanism has been identified as a camber-wake interaction that depends on the unsteady aerodynamics; the system is inherently stable if the influence of the wake is neglected. These results agree with the conclusions attained by similar studies in the context of finite cantilevered flexible plates.

Parametric studies were carried out to investigate the flutter mechanisms of systems comprising two DOF (plunge-camber and pitch-camber) and the limit for the camber-dominated instability was found to be based on frequency ratios. For the system consisting of three DOF (plunge, pitch and camber), three different behaviour patterns have been distinguished, defined by two different flutter mechanisms and static divergence. The two flutter modes have been clearly identified and correspond to plunge-camber coupling and triple-mode interaction. The influence of moving the elastic axis toward the trailing edge plays a scaling and offsetting role. However, displacing it towards the leading edge has a critical effect, since the typical dip that appears in flutter curves is eliminated.

This study has illustrated the aeroelastic instabilities that may be found on flexible airfoil designs, hitherto scarcely analyzed, and has provided simple analytical models to estimate their occurrence. In particular, it has been proved that camber bending deformations significantly alter the stability boundaries of the classical rigid systems.

The proposed methodology is readily available for extension to other cross-sections and camber shapes, as well as to different material proper- 
ties. It is also suitable for any arbitrary (small) deformations provided the airfoil is thin, since the camber line could be defined by a polynomial of any desired order, and the relevant structural properties and aerodynamic loads would be determined accordingly.

Although not as accurate as more intricate models and limited to the linear regime, this 2-D aeroelastic model can be a very useful tool for first estimations of the aeroelastic response of compliant airfoils and slender wings. The simplicity of the approach represents a great asset compared, for instance, to more computationally-expensive 3-D lifting-surface and panel methods coupled with a plate model.

\section{Acknowledgments}

Joseba Murua gratefully acknowledges the partial support of Fundación Caja Madrid and the Department of Education, Universities and Research of the Basque Government. 


\section{References}

ABAQUS, 1998. Standard Users Manual Version 5.8. Hibbit, Karlsson \& Sorensen, Inc., USA.

Abramowitz, M., Stegun, I. A., 1972. Handbook of Mathematical Functions, Ninth Edition. Dover.

Argentina, M., Mahadevan, L., Feb. 2005. Fluid-flow-induced flutter of a flag. Proceedings of the National Academy of Sciences of the United States of America 102 (6), 1829-1834.

Bak, C., Gaunaa, M., Andersen, P., Buhl, T., Hansen, P., Clemmensen, K., Moeller, R., January 2007. Wind tunnel test on wind turbine airfoil with adaptive trailing edge geometry. In: 45th AIAA Aerospace Sciences Meeting and Exhibit. AIAA Paper No 2007-1016. Reno, Nevada, USA.

Barlas, T. K., van Kuik, G. A. M., 2007. State of the art and prospectives of smart rotor control for wind turbines. Journal of Physics: Conference Series 75, 012080 (20pp).

Bergami, L., Gaunaa, M., 2009. Stability investigation of an airfoil section with active flap control. Wind Energy 12 (6).

Bernhard, A. P. F., Chopra, I., 2001. Analysis of a bending-torsion coupled actuator for a smart rotor with active blade tips. Smart Materials and Structures 10 (1), 35-52.

Bisplinghoff, R. L., Ashley, H., Halfman, R. L., 1955. Aeroelasticity. AddisonWesley. 
Campanile, L. F., Anders, S., 2005. Aerodynamic and aeroelastic amplification in adaptive belt-rib airfoils. Aerospace Science and Technology 9 (1), 55-63.

Cesnik, C. E. S., Shin, S., Wilbur, M. L., 2001. Dynamic response of active twist rotor blades. Smart Materials and Structures 10 (1), 62-76.

Gaunaa, M., 2006. Unsteady 2D potential-flow forces on a thin variable geometry airfoil undergoing arbitrary motion. Tech. Rep. Ris $\varnothing-R-1478(E N)$, Risø National Laboratory, Denmark.

Huang, L., Feb. 1995. Flutter of cantilevered plates in axial flow. Journal of Fluids and Structures 9 (2), 127-147.

Johnston, C., Mason, W., Han, C., Inman, D., 2007. Actuator-work concepts applied to unconventional aerodynamic control devices. Journal of Aircraft $44(5), 1459-68$.

Katam, V., LeBeau, R. P., Jacob, J. D., June 2005. Simulation of separation control on a morphing wing with conformal camber. In: Proceedings of the 35th AIAA Fluid Dynamics Conference and Exhibit. AIAA Paper No 2005-4880.

Katz, J., Plotkin, A., 2001. Low-Speed Aerodynamics, 2nd Edition. Cambridge Aerospace Series. Cambridge University Press.

Kerho, M., 2007. Adaptive airfoil dynamic stall control. Journal of Aircraft $44(4), 1350-1360$. 
Leishman, J. G., Nguyen, K. Q., 1989. State-space representation of unsteady airfoil behaviour. AIAA Journal 28 (5), 836-844.

Lentink, D., Mueller, U. K., Stamhuis, E. J., de Kat, R., van Gestel, W., Veldhuis, L. L. M., Henningsson, P., Hedenstroem, A., Videler, J. J., van Leeuwen, J. L., April 2007. How swifts control their glide performance with morphing wings. Nature 446, 1082-1085.

Lighthill, M. J., 1960. Note on the swimming of slender fish. Journal of Fluid Mechanics 9 (2), 305-317.

Palacios, R., Cesnik, C. E. S., April 2008a. Low-speed aeroelastic modeling of very flexible slender wings with deformable airfoils. In: Proceedings of the 49th AIAA/ASME/ASCE/AHS/ASC Structures, Structural Dynamics and Materials Conference. AIAA Paper No 2008-1995, Schaumburg, Illinois, USA.

Palacios, R., Cesnik, C. E. S., 2008b. On the one-dimensional modeling of camber bending deformations in active anisotropic slender structures. International Journal of Solids and Structures 45, 2097-2116.

Parker, H. F., 1920. The Parker variable camber wing. NACA Report No 77.

Peters, D. A., 2008. Two-dimensional incompressible unsteady airfoil theory - An overview. Journal of Fluids and Structures 24, 295-312.

Peters, D. A., Karunamoorthy, S., Cao, W., 1995. Finite state induced flow models. Part I: Two-dimensional thin airfoil. Journal of Aircraft 32 (2), $313-322$. 
Rodden, W. P., Stahl, B., 1969. A strip method for prediction of damping in subsonic wind tunnel and flight flutter tests. Journal of Aircraft 6 (1), $9-17$.

Santer, M., Pellegrino, S., 2007. Topology optimization of adaptive compliant aircraft wing leading edge. In: AIAA/ASME/ASCE/AHS/ASC Structures, Structural Dynamics and Materials Conference. AIAA Paper 20071714. Waikiki, HI, USA.

Sears, W. R., 1941. Some aspects of non-stationary airfoil theory and its practical application. Journal of the Aeronautical Sciences 8 (5), 104-108.

Spielberg, I. N., 1953. The two-dimensional incompressible aerodynamic coefficients for oscillatory changes in airfoil camber. Journal of Aeronautical Sciences 20, 432-434.

Stanford, B., Abdulrahim, M., Lind, R., Ifju, P., 5 2007. Investigation of membrane actuation for roll control of a micro air vehicle. Journal of Aircraft 44 (3), 741-749.

Tang, L., Païdoussis, M. P., Jiang, J., June 2009. The dynamics of variants of two-dimensional cantilevered flexible plates in axial flow. Journal of Sound and Vibration 323, 214-231.

Theodorsen, T., 1935. General theory of aerodynamic instability and the mechanism of flutter. NACA Report No 496.

Theodorsen, T., Garrick, I. E., 1941. Flutter calculations in three degrees of freedom. NACA Report No 741. 
Wagner, H., 1925. Über die Entstehung des Dynamischen Auftriebes von Tragflgeln. Zietschrift für Angewandte Mathematik und Mechanick 5 (1), 17.

Wu, T. Y., 1961. Swimming of a waving plate. Journal of Fluid Mechanics $10(3), 321-324$.

Wu, T. Y., 1971. Hydromechanics of swimming propulsion. Part 1. Swimming of a two-dimensional flexible plate at variable speeds in an inviscid fluid. Journal of Fluid Mechanics 46 (2), 337-355.

Zeiler, T. A., 2000. Results of Theodorsen and Garrick revisited. Journal of Aircraft. Engineering notes 37 (5), 918-920. 


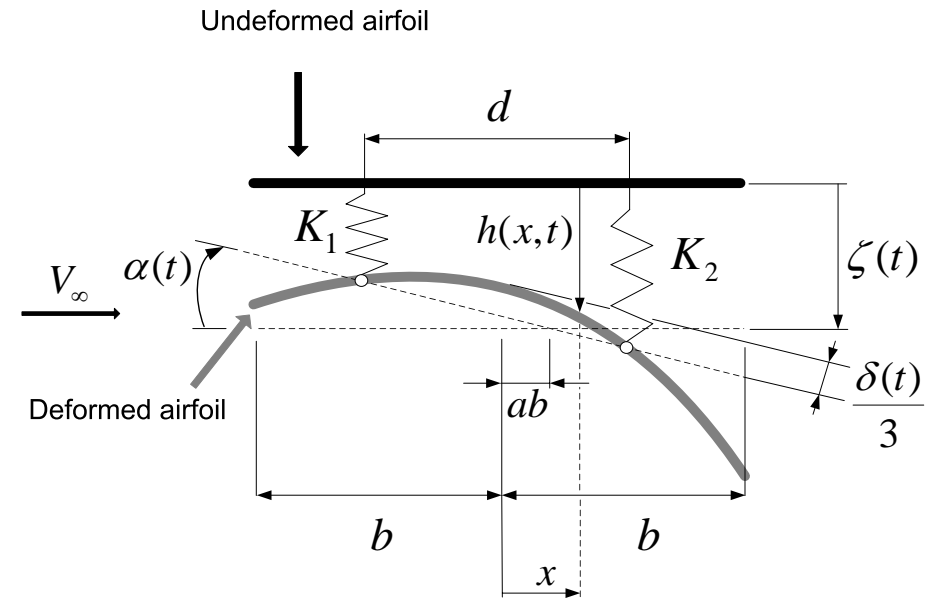

Figure 1: Airfoil camber line with two spring-supported rigid-body (plunge and pitch) and one elastic (camber deformations) degrees of freedom.

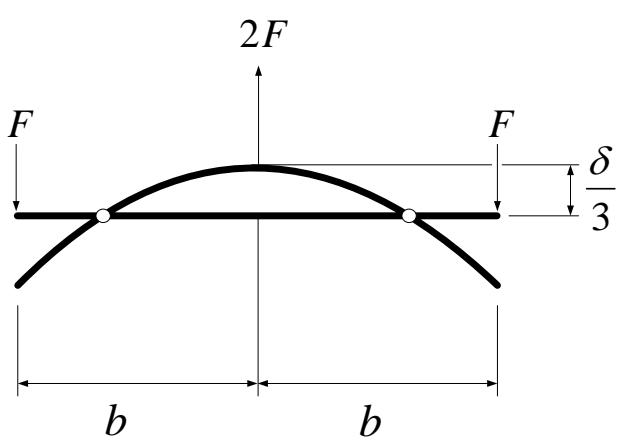

Figure 2: Cross-section of the 3-D finite-element model for the determination of the camber bending stiffness. 


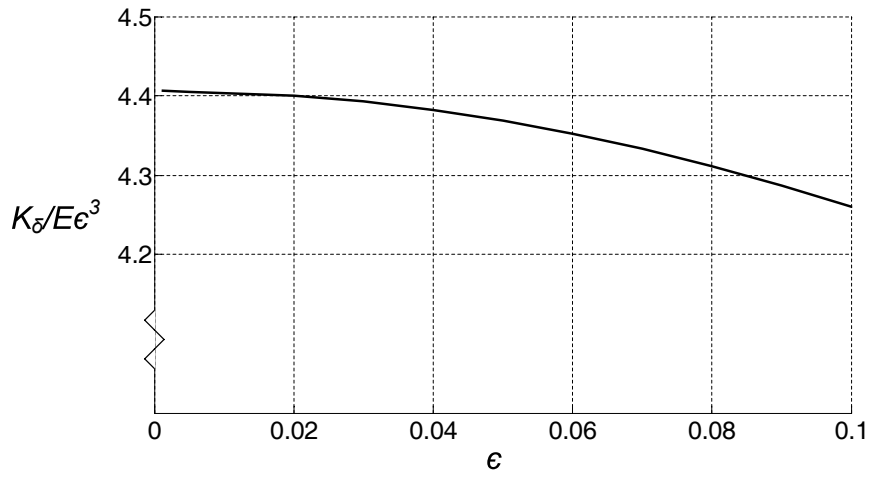

Figure 3: Normalized camber bending stiffness for a linear elastic material $(\nu=0.3)$.

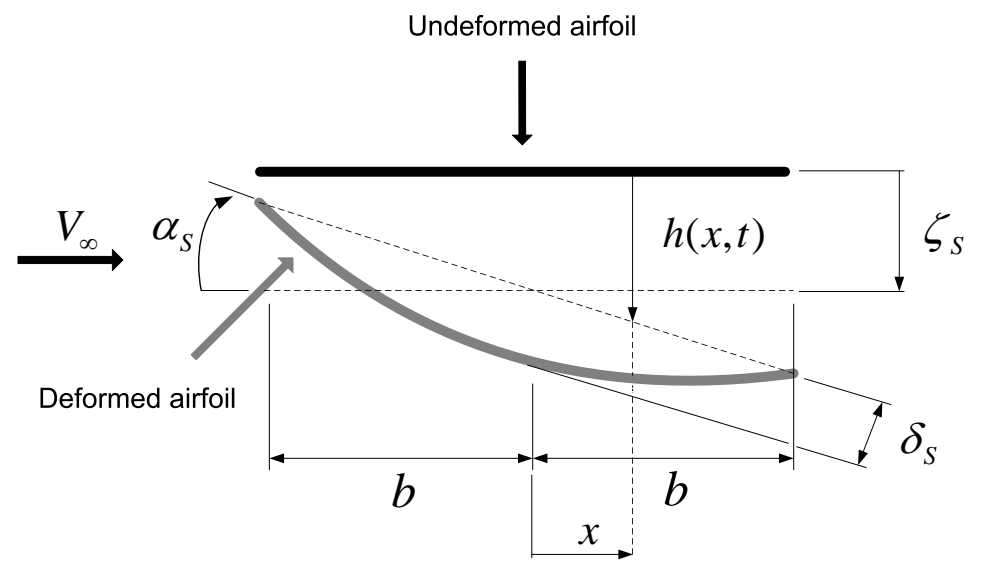

Figure 4: Spielberg's model (Spielberg, 1953) for the airfoil with plunge, pitch and camber degrees of freedom. 


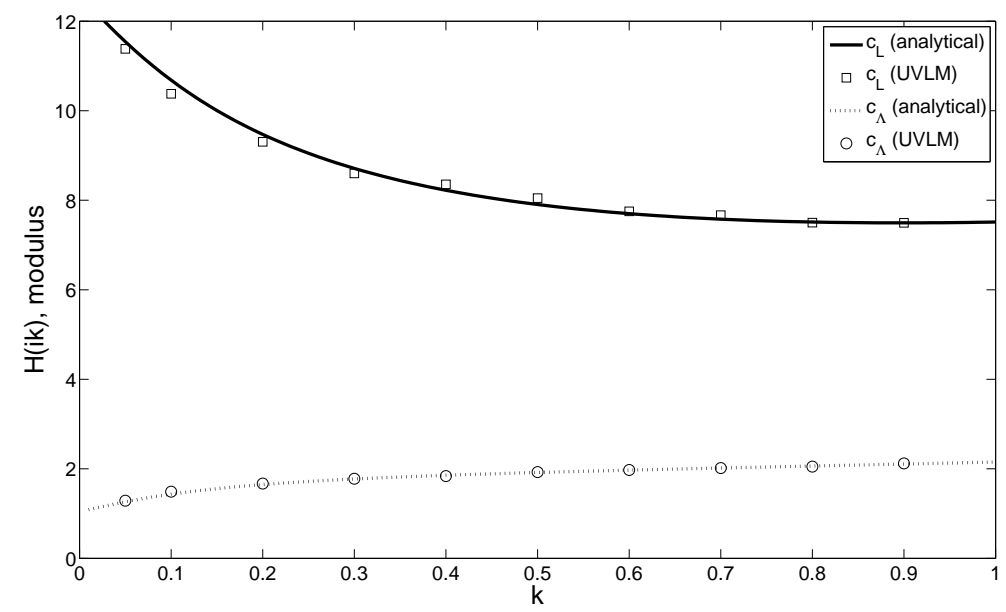

Figure 5: Modulus of the aerodynamic transfer function for the lift and bimoment coefficients under harmonic camber oscillations at different reduced frequencies.

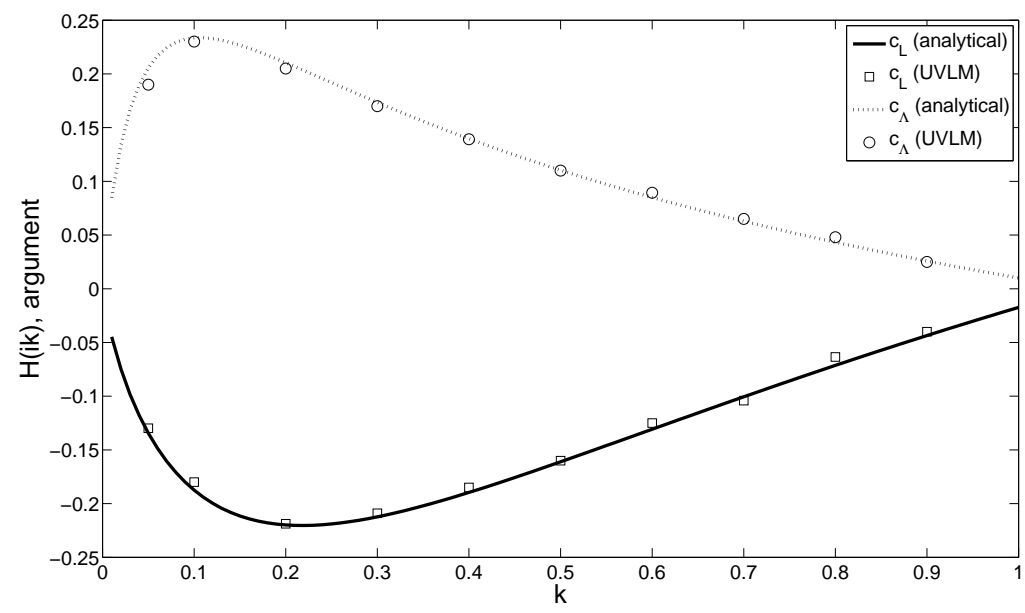

Figure 6: Argument of the aerodynamic transfer function for the lift and bimoment coefficients under harmonic camber oscillations at different reduced frequencies. 


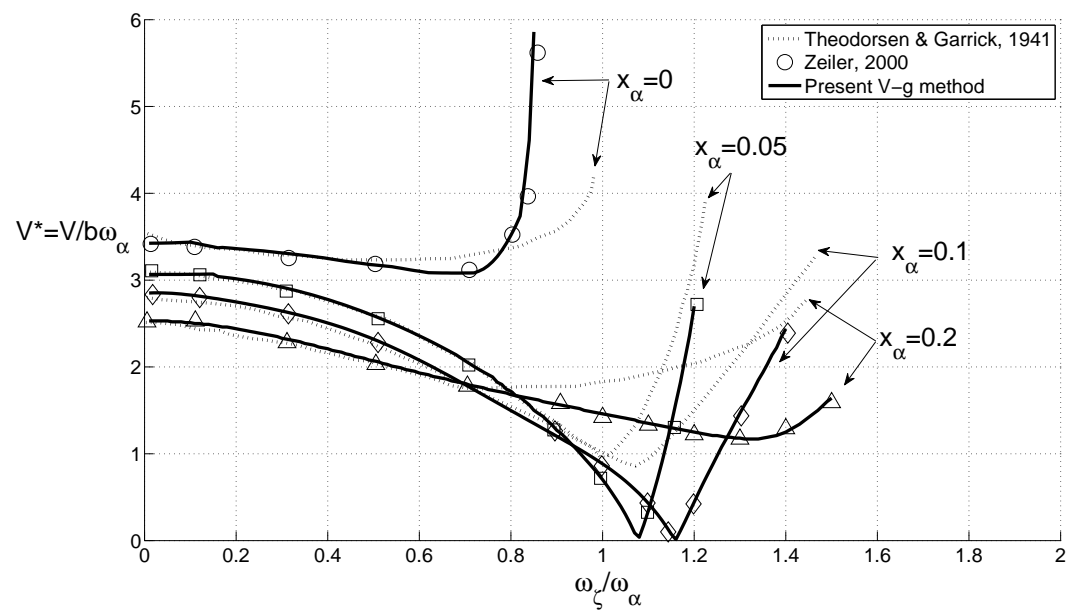

Figure 7: Validation of numerical algorithms: current solution (V-g method) compared to (Theodorsen and Garrick, 1941) and (Zeiler, 2000) $\left(a=-0.3, \kappa=0.05\right.$ and $\left.r_{\alpha}^{2}=0.25\right)$.

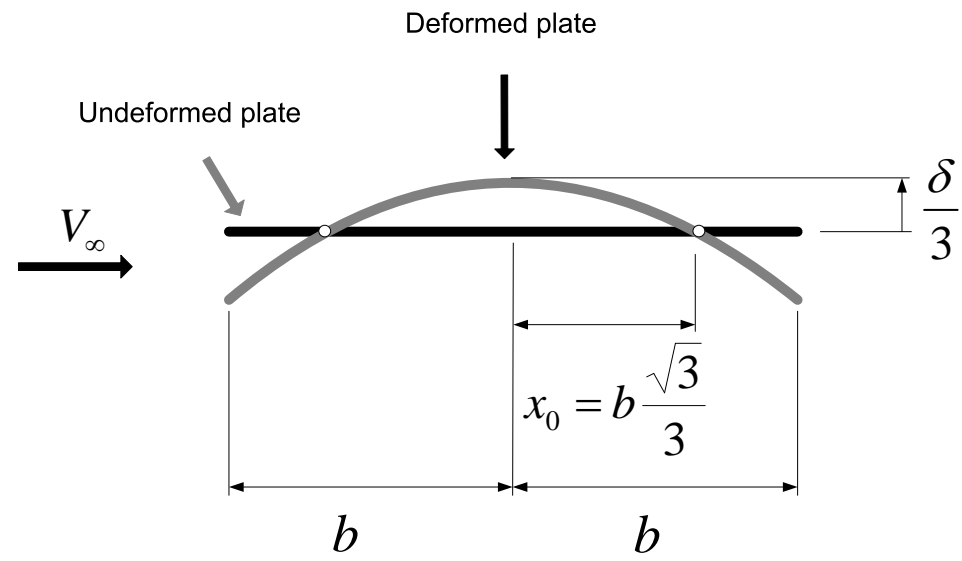

Figure 8: Single-camber system. 


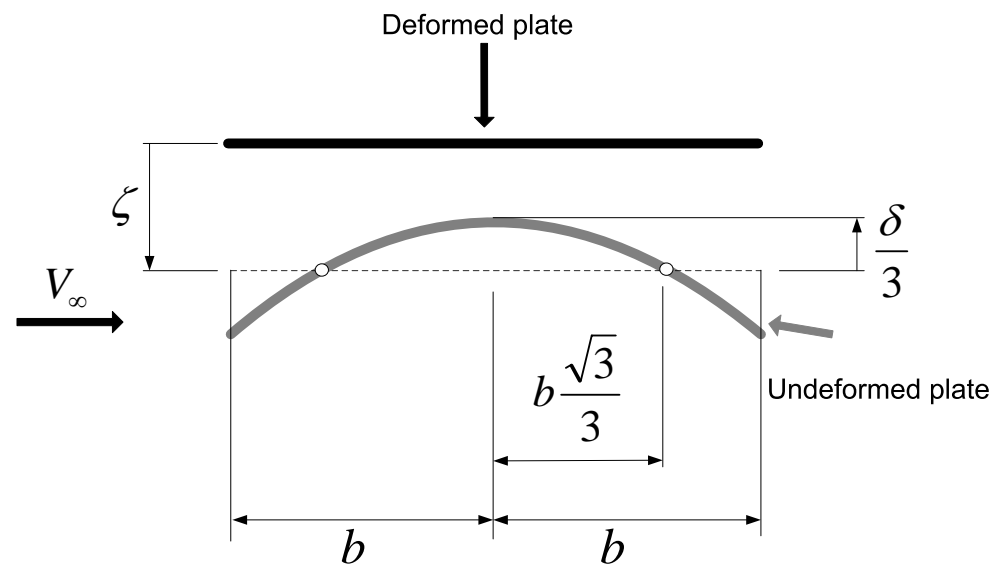

Figure 9: Plunge-camber system.

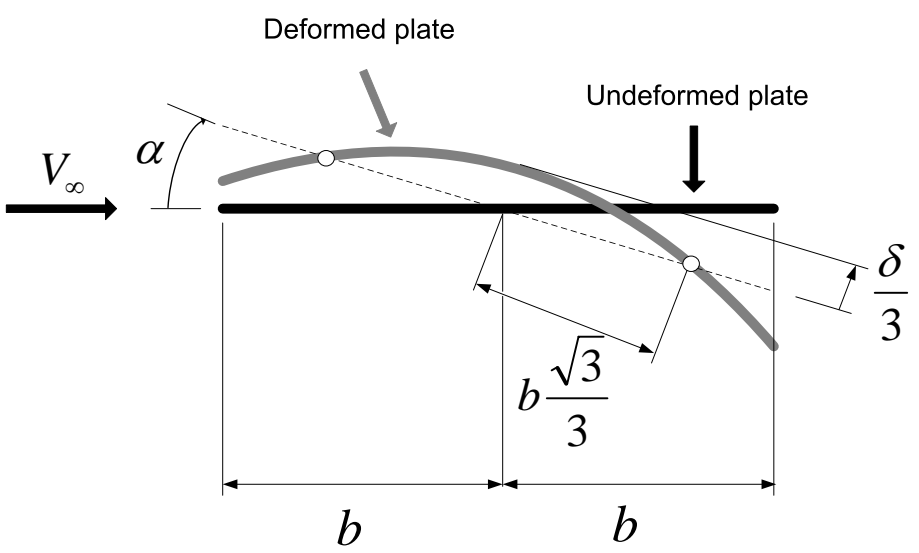

Figure 10: Pitch-camber system. 


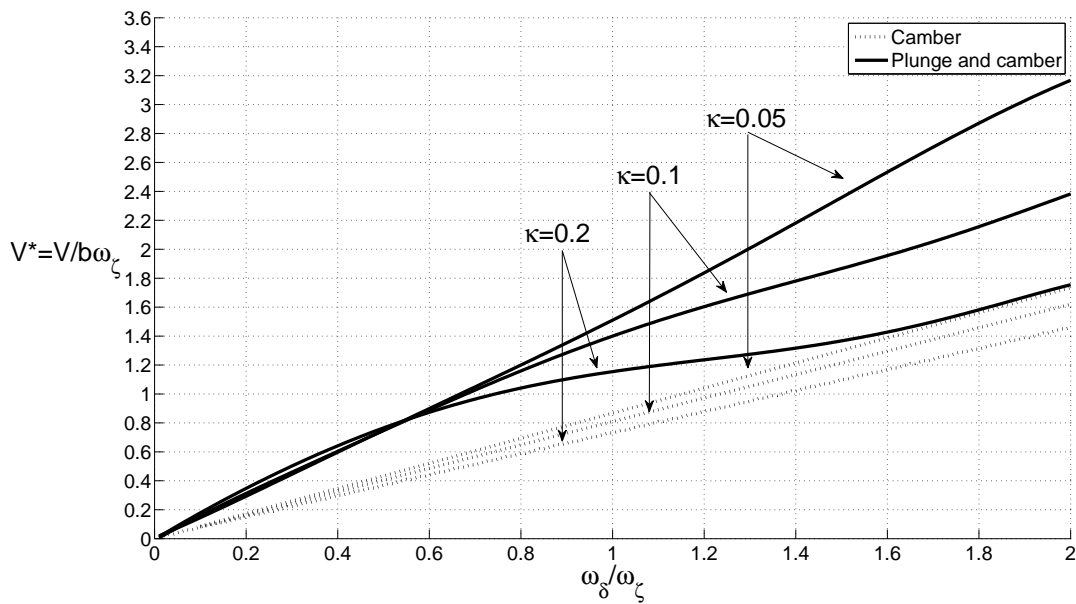

Figure 11: Flutter speed for the plunge-camber and single-camber systems $(a=0)$.

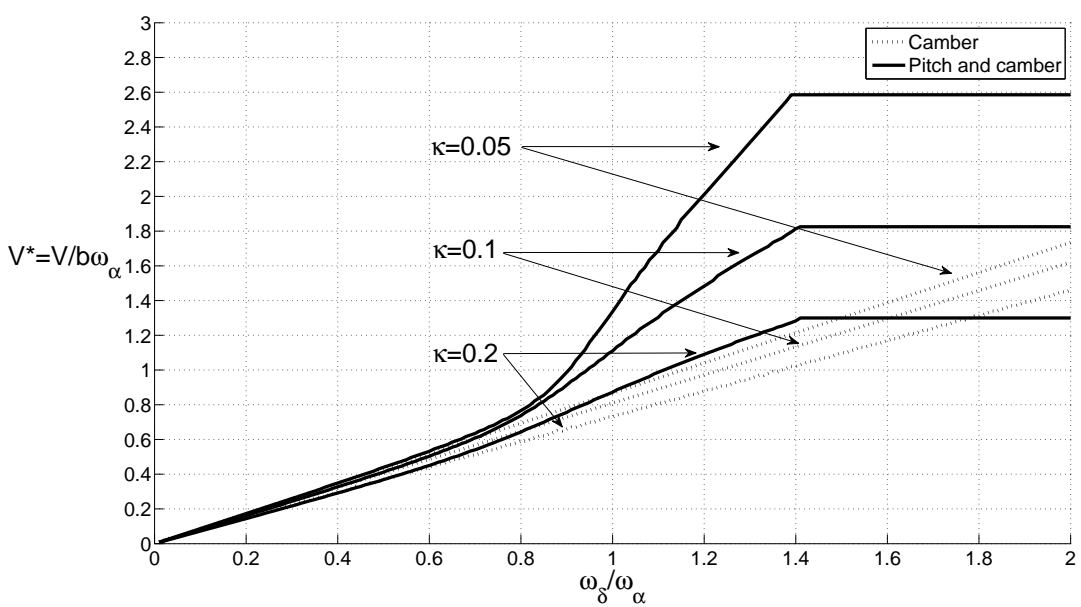

Figure 12: Flutter speed for the pitch-camber and single-camber systems $(a=0)$. 


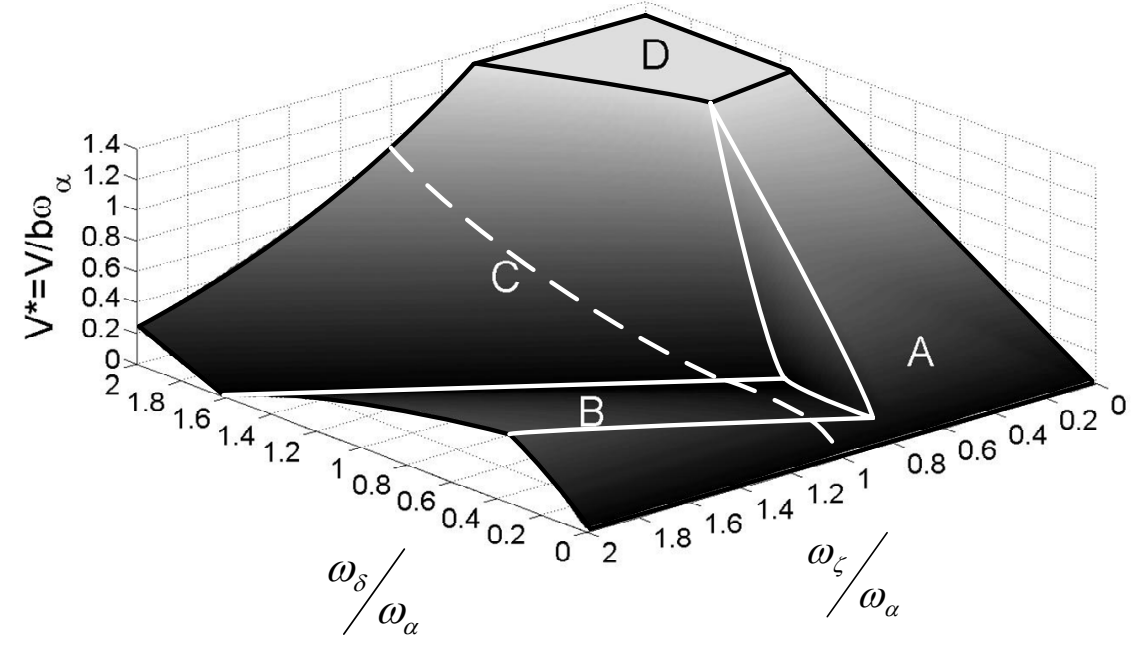

Figure 13: Flutter stability boundaries for a plunge-pitch-camber system ( $a=0$ and $\kappa=0.2)$.

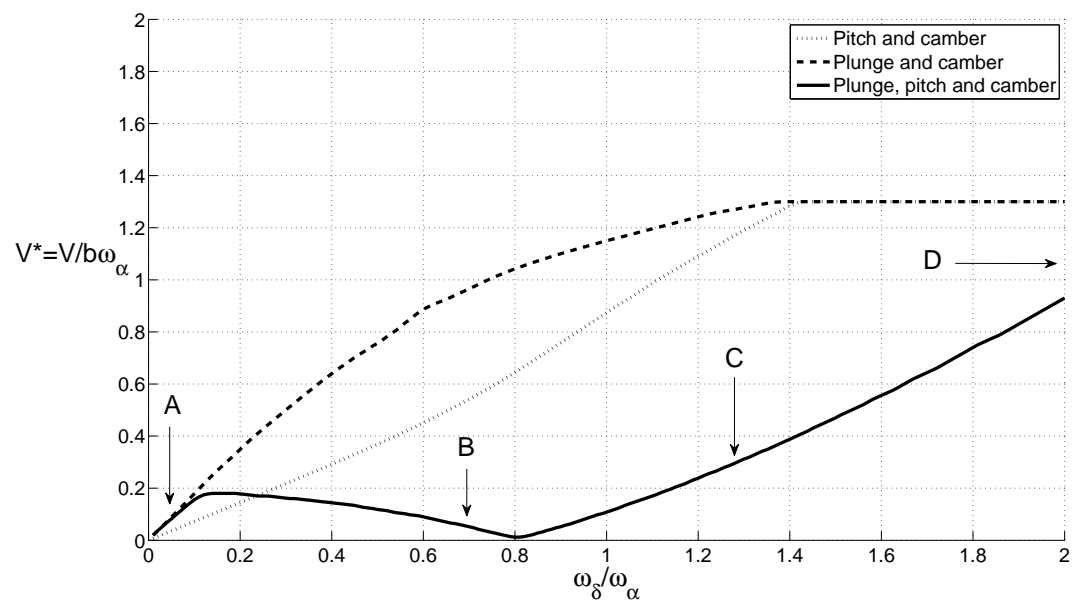

Figure 14: Flutter speed for plunge-pitch-camber, plunge-camber and pitch-camber systems $\left(\frac{\omega_{\zeta}}{\omega_{\alpha}}=1, a=0\right.$ and $\left.\kappa=0.2\right)$. 


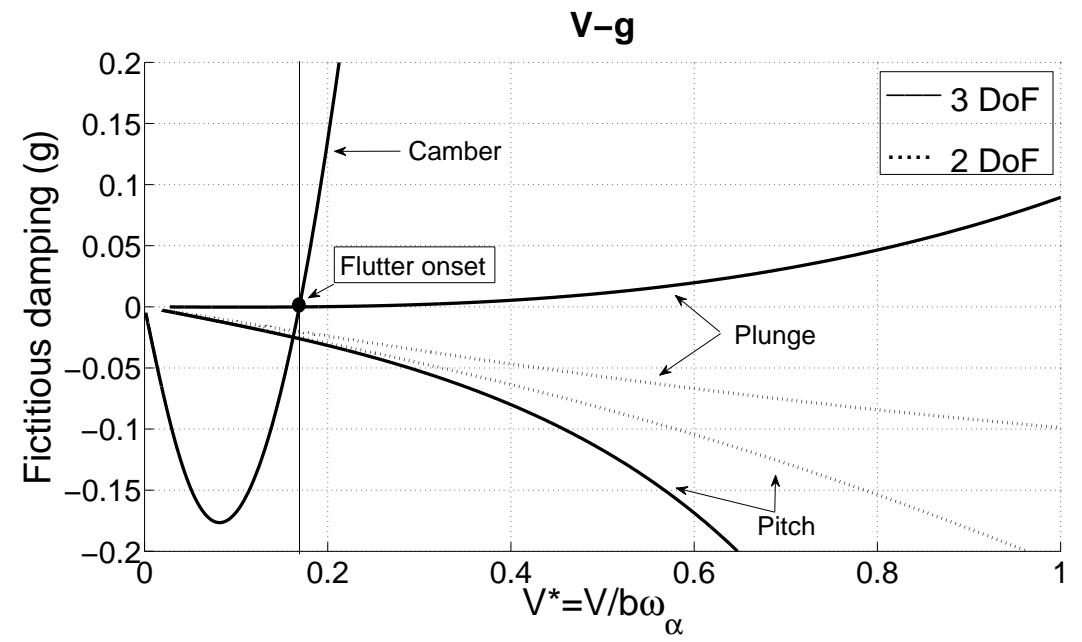

Figure 15: V-g plot for a plunge-pitch-camber (3 DOF) and a plunge-pitch (2 DOF) airfoil. Region A $\left(\frac{\omega_{\zeta}}{\omega_{\alpha}}=1, \frac{\omega_{\delta}}{\omega_{\alpha}}=0.1, a=0\right.$ and $\left.\kappa=0.2\right)$.

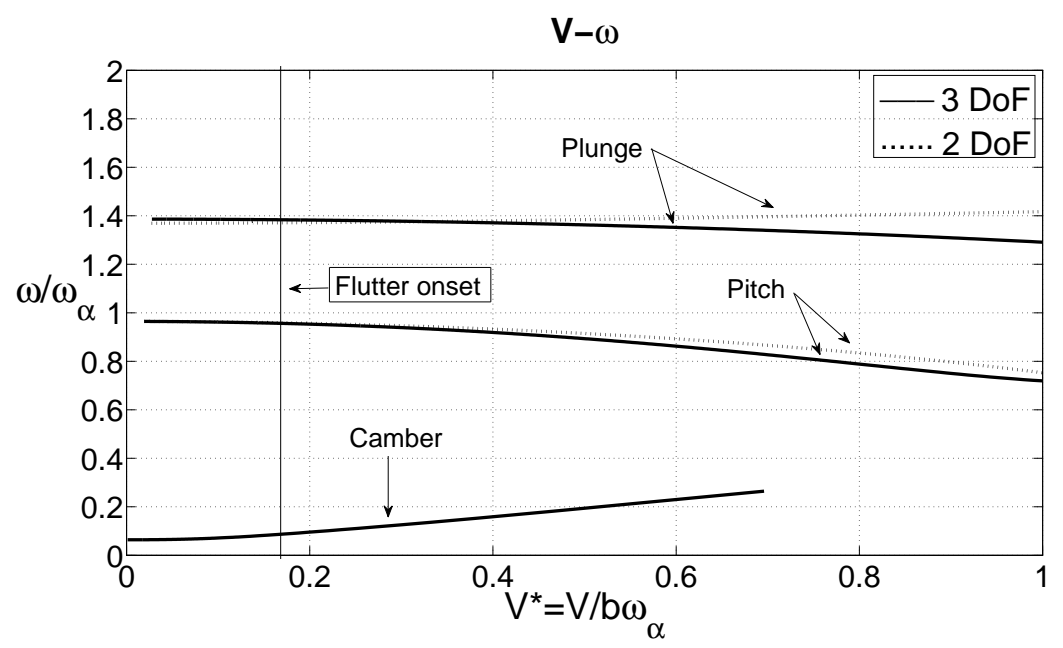

Figure 16: V- $\omega$ plot for a plunge-pitch-camber (3 DOF) and a plunge-pitch (2 DOF) airfoil. Region $\mathrm{A}\left(\frac{\omega_{\zeta}}{\omega_{\alpha}}=1, \frac{\omega_{\delta}}{\omega_{\alpha}}=0.1, a=0\right.$ and $\left.\kappa=0.2\right)$. 


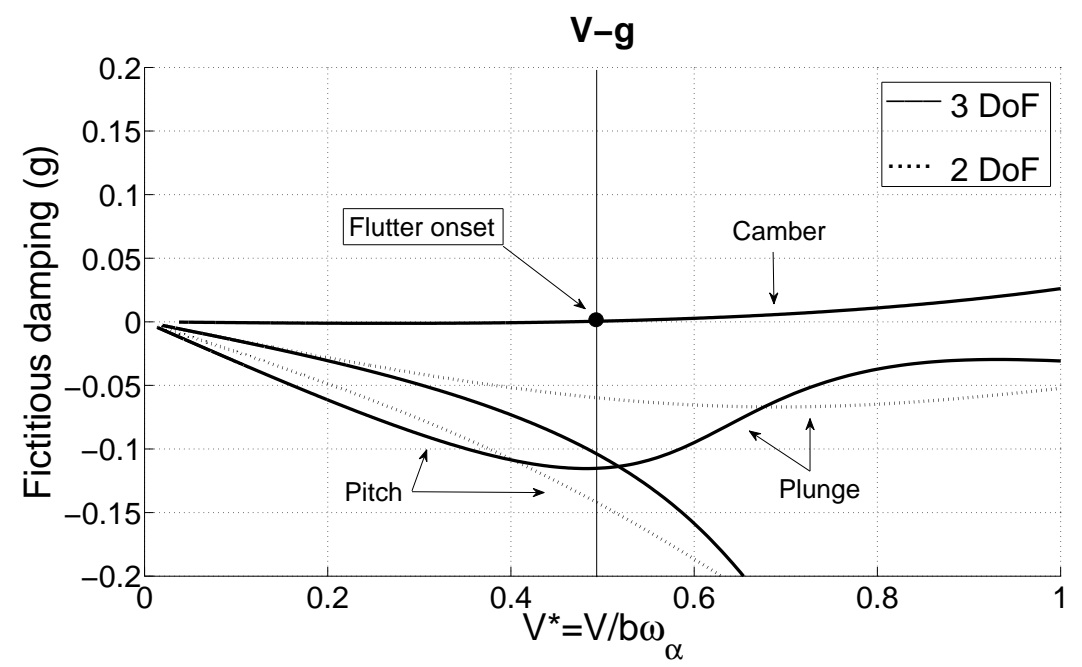

Figure 17: V-g plot for a plunge-pitch-camber (3 DOF) and a plunge-pitch (2 DOF) airfoil. Region $\mathrm{C}\left(\frac{\omega_{\zeta}}{\omega_{\alpha}}=1, \frac{\omega_{\delta}}{\omega_{\alpha}}=1.5, a=0\right.$ and $\left.\kappa=0.2\right)$.

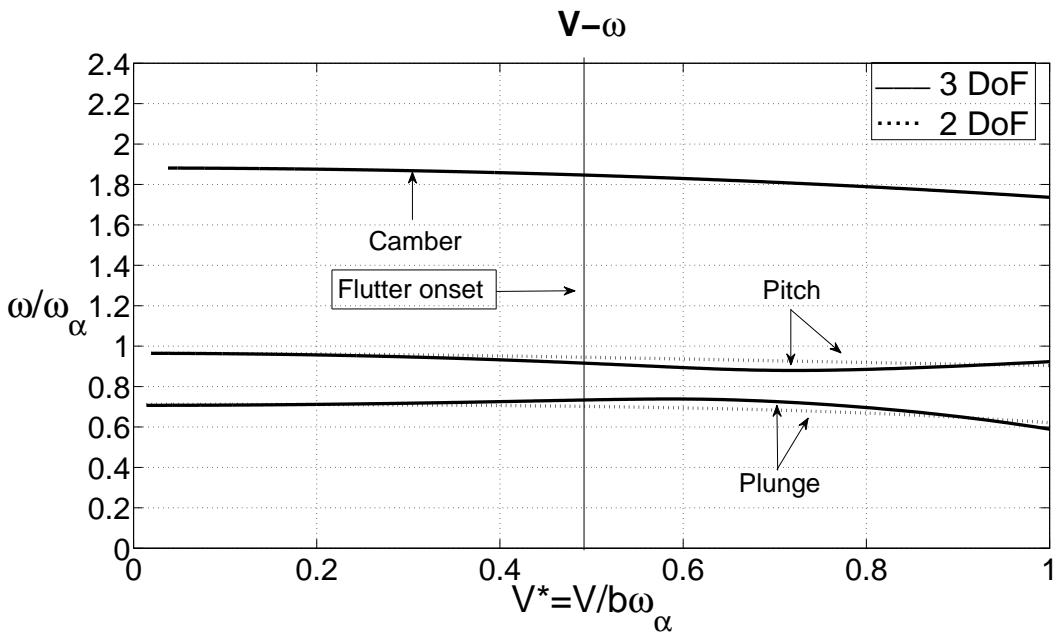

Figure 18: V- $\omega$ plot for a plunge-pitch-camber (3 DOF) and a plunge-pitch (2 DOF) airfoil. Region $\mathrm{C}\left(\frac{\omega_{\zeta}}{\omega_{\alpha}}=1, \frac{\omega_{\delta}}{\omega_{\alpha}}=1.5, a=0\right.$ and $\left.\kappa=0.2\right)$. 


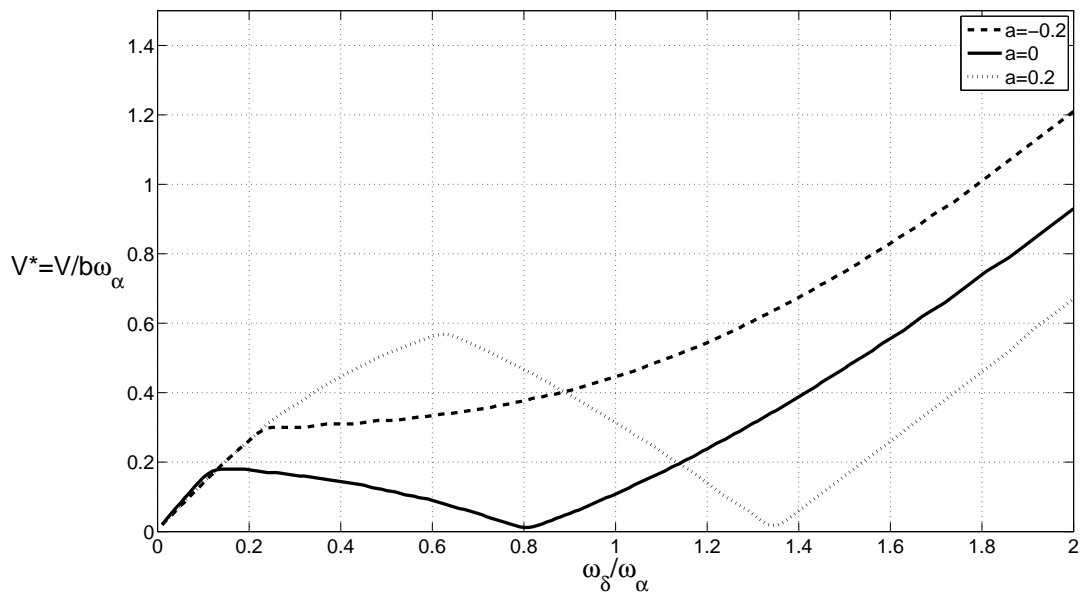

Figure 19: Influence of the location of the elastic axis, $a$, over the flutter speed of a plunge-pitch-camber (3 DOF) airfoil $\left(\frac{\omega_{\zeta}}{\omega_{\alpha}}=1\right.$ and $\left.\kappa=0.2\right)$. 


\begin{tabular}{cccccc}
\hline \hline$\frac{\omega_{\delta}}{\omega_{\alpha}}$ & $\frac{\omega_{\zeta}}{\omega_{\alpha}}$ & Region & $v_{\zeta}$ & $v_{\alpha}$ & $v_{\delta}$ \\
\hline 0.1 & 1.0 & $\mathrm{~A}$ & $0.3_{0^{\circ}}$ & $0.008_{-146^{\circ}}$ & $1_{0^{\circ}}$ \\
0.2 & 0.5 & $\mathrm{~A}$ & $0.6_{24^{\circ}}$ & $0.05_{-170^{\circ}}$ & $0.8_{0^{\circ}}$ \\
0.5 & 1.0 & $\mathrm{~B}$ & $0.2_{180^{\circ}}$ & $0.04_{87^{\circ}}$ & $1_{0^{\circ}}$ \\
0.8 & 1.0 & $\mathrm{~B} \& \mathrm{C}$ & $0.2_{180^{\circ}}$ & $0.02_{88^{\circ}}$ & $1_{0^{\circ}}$ \\
1.5 & 1.0 & $\mathrm{C}$ & $0.09_{175^{\circ}}$ & $0.09_{78^{\circ}}$ & $1_{0^{\circ}}$ \\
\hline \hline
\end{tabular}

Table 1: Eigenvectors at flutter point. Plunge, pitch and camber degrees of freedom. They are in the format $v_{i}=\lambda_{\beta}, \lambda$ representing the modulus of $v_{i}$, and $\beta$ its argument in degrees. 